\title{
On the highest energy emission from millisecond pulsars
}

\section{P. M. Saz Parkinson*}

Santa Cruz Institute for Particle Physics, University of California, Santa Cruz, CA, USA Dept. of Physics \& Laboratory for Space Research, The University of Hong Kong, Hong Kong E-mail: pablosp@hku.hk

\section{Andrea Belfiore}

Istituto di Astrofisica Spaziale e Fisica Cosmica Milano, via E. Bassini 15, 20133, Milano, Italy

\section{David Carreto Fidalgo}

Grupo de Altas Energías (GAE), Universidad Complutense, 28040 Madrid, Spain

\section{Colin J. Clark}

Albert-Einstein-Institut, Max-Planck-Institut für Gravitationsphysik, Hannover, Germany Leibniz Universität Hannover, D-30167 Hannover, Germany

Jodrell Bank Centre for Astrophysics, School of Physics and Astronomy, The University of Manchester, Manchester M13 9PL, UK

\section{Matthew Kerr}

Space Science Division, Naval Research Laboratory, Washington, DC 20375-5352, USA

\section{Lars Nieder}

Albert-Einstein-Institut, Max-Planck-Institut für Gravitationsphysik, Hannover, Germany Leibniz Universität Hannover, D-30167 Hannover, Germany

\section{on behalf of the Fermi LAT Collaboration}

Fermi has detected over 200 pulsars above $100 \mathrm{MeV}$. In a previous work, using 3 years of LAT data (1FHL catalog) we reported that 28 of these pulsars show emission above $10 \mathrm{GeV}$; only three of these, however, were millisecond pulsars (MSPs). The recently-released Third Catalog of Hard Fermi-LAT Sources (3FHL) contains over 1500 sources showing emission above $10 \mathrm{GeV}, 17$ of which are associated with gamma-ray MSPs. Using three times as much data as in our previous study (1FHL), we report on a systematic analysis of these pulsars to determine the highest energy (pulsed) emission from MSPs and discuss the best possible candidates for follow-up observations with ground-based TeV instruments (H.E.S.S., MAGIC, VERITAS, and the upcoming CTA).

7th Fermi Symposium 2017

15-20 October 2017

Garmisch-Partenkirchen, Germany

\footnotetext{
*Speaker.
} 


\section{Introduction}

Studies of the $\gamma$-ray sky above $10 \mathrm{GeV}$ were limited in the past by the relatively poor sensitivity of the instruments. The Energetic Gamma Ray Experiment Telescope (EGRET) on the Compton Gamma Ray Observatory (CGRO) detected just over 1500 photons above $10 \mathrm{GeV}$ in its 9-year lifetime, 37 of which fell within $1^{\circ}$ of one of the 5 EGRET-detected $\gamma$-ray pulsars known at the time [1]. The Fermi Large Area Telescope (LAT), launched in 2008, has already exceeded EGRET's lifetime and with its larger field of view and better sensitivity, has collected about three orders of magnitude more photons than EGRET did. Based on silicon-strip detector technology, compared to the older generation gas-based spark chambers, the LAT's improved sensitivity is particularly significant at the highest energies. The First Fermi-LAT Catalog of sources above $10 \mathrm{GeV}$ (1FHL) [2], using the first three years of LAT data above $10 \mathrm{GeV}$, contained 514 sources. Most of the 1FHL sources (76\%) are associated with active galactic nuclei, but 27 are associated with pulsars, while $\sim 13 \%$ of $1 \mathrm{FHL}$ sources are unassociated.

\section{Pulsars in 1FHL and 2FHL}

The 27 pulsars coincident with 1FHL sources covered every category, including the 5 EGRETdetected pulsars, roughly equal numbers of young radio loud and radio quiet pulsars, and 5 millisecond pulsars (MSPs). A likelihood ratio analysis was carried out, comparing the low energy $(>100 \mathrm{MeV})$ light curve to the high energy $(>10 \mathrm{GeV})$ events, in order to determine whether significant pulsations were detected above $10 \mathrm{GeV}$ and $25 \mathrm{GeV}$. A majority of the pulsars tested $\left(20 / 25^{1}\right)$ were found to "pulse" above $10 \mathrm{GeV}$, with 12 pulsars showing significant pulsations above $25 \mathrm{GeV}$ (including the MSP J0614-3329). In addition to these, 14 pulsars from the 2nd Pulsar Catalog which showed evidence for possible emission above $10 \mathrm{GeV}$ were studied. Of these, eight showed significant pulsations above $10 \mathrm{GeV}$, including the MSPs J2017+0603 and J2302+4442. In short, [2] showed that at least 28 gamma-ray pulsars show pulsations above $10 \mathrm{GeV}, 5$ of which were MSPs, with one MSP (J0614-3329) showing pulsations above $25 \mathrm{GeV}$. The Second Catalog of Hard Fermi-LAT Sources (2FHL) [3] spanned 80 months and used the improved Pass 8 data. However, the low energy threshold this time around was set at $50 \mathrm{GeV}$, instead of $10 \mathrm{GeV}$ used for 1FHL. This resulted in far fewer sources (360), and only one of these was coincident with a pulsar (Vela). Indeed, [4] confirmed that Vela emits pulsations above $50 \mathrm{GeV}$, something also confirmed now by H.E.S.S. [5].

\section{The 3FHL Catalog}

The Third Catalog of Hard Fermi-LAT Sources (3FHL) [6] uses 7 years of Pass 8 data above $10 \mathrm{GeV}$ and contains 1556 sources. It's interesting to note that the number of $\gamma$-ray sources above $10 \mathrm{GeV}$ now slightly exceeds the number of $>10 \mathrm{GeV}$ photons detected by EGRET in its 9-year lifetime. Like the 1FHL before it, the 3FHL contains mainly (79\%) sources associated with active galactic nuclei, with $\sim 13 \%$ of its sources being unassociated.

${ }^{1}$ PSR J1536-4948 and J2339-0533 were not part of the Second Fermi LAT Catalog of Gamma-ray Pulsars (2PC, [7]), so these were left out of the analysis. 


\section{MSPs in 3FHL}

Compared to 1FHL, there are approximately three times the number of pulsars associated with 3FHL sources, including $17 \gamma$-ray MSPs. Note that only 5 MSPs were previously coincident with 1FHL sources. We have undertaken a similar analysis to that carried out in 1FHL, but this time focussed only on the MSPs associated with 3FHL sources.

The 17 MSPs coincident with 3FHL sources span a range of different categories of MSPs: isolated MSPs such as J0340+4130 and J0533+6759, the Black Widow pulsar J1311-3430, Redback MSPs, such as J2215+5135 and J2339-0533, and even the transitional MSP J1227-4853. We carried out a dedicated analysis using 9 years of LAT data (three times the amount of data used in 1FHL). The improved statistics enabled us to generate the low energy pulse profile of each pulsar using events above $1 \mathrm{GeV}$. Because the pulse profile of most pulsars is known to change with energy (with pulses typically becoming narrower), this improves the sensitivity of the pulsation search at higher energies. We performed a spectral analysis of each of the pulsars, followed by an updated timing analysis of all systems. We excluded J1227-4853 from our analysis for now, since its pulsed emission is variable and requires a more careful dedicated analysis.

We find that all 16 MSPs that we tested showed significant pulsations above $10 \mathrm{GeV}$. This includes PSRs J1536-4948 and J2339-0533 which had been found to be coincident with 1FHL sources but were not part of the previous analysis as these pulsars were not part of 2PC [7]. Of these 16 MSPs, five show significant pulsations above 25 GeV: J0218+4232, J0614-3329, J12311411, J1311-3430, and J1536-4948 [8].

PSR J0218+4232 was discovered over 25 years ago [9]. A tentative detection was claimed using EGRET data [11] and it was among the first MSPs to be firmly detected by the Fermi LAT [10]. Its spindown luminosity of $2.4 \times 10^{35} \mathrm{erg} \mathrm{s}^{-1}$ makes it the second most energetic field $\mathrm{MSP}^{2}$ (after J1939+2134), of the $\sim 100$ gamma-ray MSPs detected so far by Fermi. Its non-thermal X-ray flux, as well as its radio luminosity $\left(L_{400}=S_{400} d_{k p c}^{2} \sim 400 \mathrm{mJy} \mathrm{kpc}^{2}\right)$ are among the highest of all MSPs [7]. J0614-3329 and PSR J1231-1411 were two of the first three MSPs discovered in radio searches of pulsar-like unassociated $\gamma$-ray sources [12] and as such are among the brightest and most energetic MSPs detected by the LAT $\left(\sim 2 \times 10^{34} \mathrm{erg} \mathrm{s}^{-1}\right)$. J0614-3329 was the only MSP which was previously (in 1FHL) shown to have significant pulsations above $25 \mathrm{GeV}$; in fact, despite being due to a single photon, the maximum energy above which the pulsed emission was still found significant for this pulsar exceeded $60 \mathrm{GeV}$. Recently, [13] reported emission from this pulsar up to $60 \mathrm{GeV}$. The Black Widow pulsar J1311-3430 was the first binary MSP discovered in blind searches of LAT data [14]. Finally, PSR J1536-4948 was discovered by the Giant Metrewave Radio Telescope (GMRT), in its searches of pulsar-like LAT $\gamma$-ray sources [15].

It is indicative of the success of Fermi, that 4 out of the $5 \gamma$-ray MSPs now known to be emitting above $25 \mathrm{GeV}$ were not even known prior to the launch of Fermi ${ }^{3}$.

\section{VHE Pulsars}

At energies above $50 \mathrm{GeV}$, the LAT unfortunately is simply too small to collect sufficient

\footnotetext{
2 i.e. not in a Globular Cluster.

${ }^{3}$ J0614-3329, however, is coincident with an EGRET unidentified source.
} 
statistics. Indeed, the total exposure on each of the 16 MSPs described above, over the 9-year period of observation, ranges from $\sim 1.0-1.7 \mathrm{~m}^{2} \mathrm{yr}$, depending on the location in the sky. Fortunately, ground-based $\gamma$-ray telescopes have made tremendous advances in recent years. The MAGIC telescope led the way with the first detection of the Crab pulsar above $25 \mathrm{GeV}$ [16], followed by the VERITAS detection of pulsed emission above $100 \mathrm{GeV}$ [17]. Most recently, MAGIC has detected the Crab pulsar up to $\mathrm{TeV}$ energies [18]. Added to the H.E.S.S. detection of the Vela pulsar up to $120 \mathrm{GeV}$ [5], we now have two pulsars that emit at Very High Energies (VHE, >100 GeV). A number of models have been developed to explain this emission (e.g. [19, 20, 21, 22]). Observationally, it remains to be seen whether any other pulsars can be found that emit at these energies. Current instruments like H.E.S.S., MAGIC, and VERITAS continue to search for such VHE pulsars, and in a few years, the much more sensitive Cherenkov Telescope Array (CTA) will join the excitement, likely detecting a number of $\gamma$-ray pulsars, starting at a few tens of $\mathrm{GeV}$ [23].

\section{Summary}

The 1FHL Catalog [2], based on 3 years of LAT data above $10 \mathrm{GeV}$ showed that 28 (13) pulsars showed significant pulsations above 10 (25) GeV, including 5 (1) MSPs emitting above 10 (25) GeV. The 3FHL catalog includes 17 sources coincident with MSPs. We carried out an analysis of 16 of these (excluding the transitional system J1227-4853) and found that all 16 of them show significant pulsations above $10 \mathrm{GeV}$, with 5 of them showing significant pulsations above $25 \mathrm{GeV}$. Detailed results are presented in [8]. Future obvservations with ground-based $\gamma$-ray telescopes like H.E.S.S., MAGIC, and VERITAS, and in the near future CTA, will determine whether any of these systems are VHE pulsars, emitting above $100 \mathrm{GeV}$, like the Crab and Vela. The detection of new pulsars at these energies will be crucial to test the many models that have been developed in recent years to explain the VHE emission of the Crab and (to a lesser extent) Vela pulsars.

\section{Acknowledgments}

The Fermi-LAT Collaboration acknowledges support for LAT development, operation and data analysis from NASA and DOE (United States), CEA/Irfu and IN2P3/CNRS (France), ASI and INFN (Italy), MEXT, KEK, and JAXA (Japan), and the K.A. Wallenberg Foundation, the Swedish Research Council and the National Space Board (Sweden). Science analysis support in the operations phase from INAF (Italy) and CNES (France) is also gratefully acknowledged. This work performed in part under DOE Contract DE-AC02-76SF00515. This work was supported by the National Aeronautics and Space Administration (NASA) through the Fermi Guest Investigator grant NNX15AW43G. This research was partially carried out using the HKU Information Technology Services research computing facilities that are supported in part by the Hong Kong UGC Special Equipment Grant (SEG HKU09).

\section{References}

[1] D. J. Thompson, D. .L. Bertsch, \& R. H. O’Neal, Jr. 2005, The Highest-Energy Photons Seen by the Energetic Gamma Ray Experiment Telescope (EGRET) on the Compton Gamma Ray Observatory', ApJS, 157, 324 
[2] M. Ackermann et al. 2013 (Fermi LAT Collaboration), The First Fermi-LAT Catalog of Sources above $10 \mathrm{GeV}, \mathrm{ApJS}, 209,34$

[3] M. Ackermann et al. 2016 (Fermi LAT Collaboration), 2FHL: The Second Catalog of Hard Fermi-LAT Sources, ApJS, 222, 5

[4] G. C. K. Leung, J. Takata, C. W. Ng et al. 2014, Fermi-LAT Detection of Pulsed Gamma-Rays above 50 GeV from the Vela Pulsar', ApJL, 797, L13

[5] A. Djannati-Ataï et al. 2017 (HESS Collaboration), Probing Vela pulsar down to 20 GeV with H.E.S.S. II observations, 6th International Symposium on High Energy Gamma-Ray Astronomy, 1792, 040028

[6] M. Ajello et al. 2017 (Fermi LAT Collaboration), 3FHL: The Third Catalog of Hard Fermi-LAT Sources, ApJS, 232, 18

[7] A. A. Abdo et al. 2013 (Fermi LAT Collaboration), The Second Fermi Large Area Telescope Catalog of Gamma-Ray Pulsars, ApJS, 208, 17

[8] P. M. Saz Parkinson et al. 2018, On the highest energy emission from Millisecond Pulsars, ApJ, in preparation

[9] J. Navarro, A. G. de Bruyn, D. A. Frail, S. R. Kulkarni, \& A. G. Lyne 1995, A Very Luminous Binary Millisecond Pulsar, ApJL, 455, L55

[10] A. A. Abdo et al. 2009 (LAT Collaboration), A Population of Gamma-Ray Millisecond Pulsars Seen with the Fermi Large Area Telescope, Science, 325, 848

[11] L. Kuiper, W. Hermsen, F. Verbunt et al. 2000, The likely detection of pulsed high-energy gamma-ray emission from millisecond pulsar PSR J0218+4232, A\&A, 359, 615

[12] S. M. Ransom et al. 2011, Three Millisecond Pulsars in Fermi LAT Unassociated Bright Sources, ApJL, 727, L16

[13] Y. Xing \& Z. Wang 2016, Fermi Study of Gamma-ray Millisecond Pulsars: the Spectral Shape and Pulsed Emission from J0614-3329 up to $60 \mathrm{GeV}, \mathrm{ApJ}, \mathbf{8 3 1}, 143$

[14] H. J. Pletsch, L. Guillemot, H. Fehrmann et al. 2012, Binary Millisecond Pulsar Discovery via Gamma-Ray Pulsations, Science, 338, 1314

[15] P. S. Ray et al. 2012, Radio Searches of Fermi LAT Sources and Blind Search Pulsars: The Fermi Pulsar Search Consortium, 2011 Fermi Symposium proceedings - eConf C110509: arXiv:1205.3089

[16] E. Aliu et al. 2008 (MAGIC Collaboration), Observation of Pulsed Gamma-Rays Above 25 GeV from the Crab Pulsar with MAGIC, Science, 322, 1221

[17] E. Aliu et al. 2011 (VERITAS Collaboration), Detection of Pulsed Gamma Rays Above $100 \mathrm{GeV}$ from the Crab Pulsar, Science, 334, 69

[18] S. Ansoldi et al. 2016 (MAGIC Collaboration), Teraelectronvolt pulsed emission from the Crab Pulsar detected by MAGIC, A\&A, 585, A133

[19] F. A. Aharonian, S. V. Bogovalov, \& D. Khangulyan, 2012, Abrupt acceleration of a cold ultrarelativistic wind from the Crab pulsar, Nature, 482, 507âĂŞ509

[20] W. Bednarek, 2012, On the origin of sub-TeV gamma-ray pulsed emission from rotating neutron stars, MNRAS, 424, 2079,

[21] M. Lyutikov, N. Otte, \& A. McCann 2012, The very high energy emission from pulsars: A case for Inverse Compton scattering, ApJ, 754, 33 
[22] A. K. Harding \& C. Kalapotharakos 2015, Synchrotron Self-Compton Emission from the Crab and Other Pulsars, ApJ, 811, 63

[23] A. Burtovoi, T. Y. Saito, L. Zampieri, \& T. Hassan 2017, Prospects for the detection of high-energy (E>25 GeV) Fermi pulsars with the Cherenkov Telescope Array, MNRAS, 471, 431 